\title{
II bis. - UN CAS DE DIGESTIBILITE PERTURBEe CHEZ LE PORC PAR LA PRESENCE EXCESSIVE DE CHLORURE DE SODIUM DANS UN ENSILAgE DE POMMES
}

PAR

\author{
G. CHARLET-LERY, A. M. LEROY, S. Z. ZELTER
}

Cette communication concerne une observation faite incidemment au cours de l'étude de la digestibilité d'un ensilage de pomme sur le porc; elle ne présente de l'intérêt que par les renseignements qu'elle apporte dans le domaine de la tolérance manifestée par cet animal envers le chlorure de sodium.

On sait que le sel accélère le péristaltisme. du tube digestif et qu'il franchit aisément la barrière intestinale, ce qui modifie vraisemblablement l'absorption digestive. La consommation de doses approchant un gramme par $\mathrm{kg}$ de poids vif risque, en outre, d'exercer chez le porc des effets toxiques.

Or, dans un précédent mémoire (voir mémoire I) il a été signalé un enrichissement considérable en chlorures des pommes conservées par macération dans une solution aqueuse renfermant $6 \mathrm{p}$. Ioo de $\mathrm{NaCl}$. Il était donc intéressant d'observer les répercussions de l'ingestion d'un tel ensilage sur son utilisation digestive par le porc, réputé être un excellent utilisateur d'aliments glucidiques.

L'observation a été enregistrée sur 3 sujets mâles Iarge White, exempts de parasites intestinaux, âgés de 4 mois $\mathrm{I} / 2$ et pesant initialement entre 35,5 et $47,5 \mathrm{~kg}$. Leur poids final, après 2 mois d'observation, oscillait entre 52,0 et $65,6 \mathrm{~kg}$.

L'aliment de référence, sous forme de farine, constitué de : orge $75 \mathrm{p}$. Ioo, levure de distillerie $5 \mathrm{p}$. Ioo, farine de viande $5 \mathrm{p}$. Ioo, farine de poisson 5 p. Ioo, tourteau d'arachide 7 p. Ioo, mélange minéral 3 p. IOo $\left(^{1}\right)$ était celui utilisé pour l'expérience d'alimentation comparée (voir mémoire III).

Les caractéristiques de l'ensilage de pommes expérimenté, particulièrement riche en chlorure, sont indiquées dans le mémoire I.

(1) Composition centésimale du mélange minéral : poudre d'os dégélatiné 40 - craie lavée $32-$ sel marin 20 - sulfate de magnésie 3,5 - carbonate de magnésie $4,2-$ sulfate ferreux 0,28 - sulfate de cuivre 0.02 . 
ment glucidique, soit significativement plus faible chez le porc que chez le ruminant :

$79,7 \pm 3,8$ I contre $90,6 \pm 0,82$, ceci pour des utilisations digestives de matières sèches sensiblement équivalentes : 86,0 $\pm 4,0$ contre $87,9 \pm \mathbf{I}, 2$.

L'explication de ce résultat paradoxal à priori que l'on pourrait suggérer, serait la suivante : nos animaux d'expérience ont absorbé

\section{TABLEAU II}

Coefficients moyens d'utilisation digestive des divers constituants des régimes.

\begin{tabular}{|c|c|c|c|}
\hline & $\begin{array}{c}\text { Période Témoin } \\
\text { (Régime de référence } \\
100 \% \text { ) }\end{array}$ & $\begin{array}{c}\text { Expérience I } \\
\text { (9 } \% \text { pommes ensilées) }\end{array}$ & $\begin{array}{c}\text { Expérience II } \\
(26 \% \text { pommes ensilées })\end{array}$ \\
\hline $\begin{array}{l}\text { Matière sèche } \ldots \ldots \ldots \ldots \\
\text { Matière organique } \ldots \ldots \ldots \\
\text { Azote } \ldots \ldots \ldots \ldots \ldots \\
\text { Matière grasse } \ldots \ldots \ldots \ldots \\
\text { Cellulose. } \ldots \ldots \ldots \ldots \ldots \\
\text { Extractifs non azotés } \ldots \ldots\end{array}$ & $\begin{array}{l}76,6 \\
79,7 \\
78,9 \\
6,9 \\
13,8 \\
87,0\end{array}$ & $\begin{array}{l}77,5 \\
79,6 \\
74,2 \\
64,4 \\
22,7 \\
87,1\end{array}$ & $\begin{array}{l}75,6 \\
79,9 \\
63,0 \\
49,8 \\
37,2 \\
89,0\end{array}$ \\
\hline
\end{tabular}

suivant le pourcentage d'ensilage de pomme inclus dans le régime, $70 \mathrm{~g}$ au moins et r75 $\mathrm{g}$ au plus de chlorure de sodium par roo $\mathrm{kg}$ de poids vif, quantités dépassant très fortement les doses de sel convenant au porc [ Io à $I 5 \mathrm{~g}$ par Ioo $\mathrm{kg}$ de poids vif $(\mathrm{I}, 2)$ soit 0,25 à 0,40 p. roo de la matière sèche ingérée (3)] ; cet excès de sel en accélérant le transit du

\section{TABLEAU III}

\section{Coefficients individuels d'utilisation digestive}

des pommes ensilées (calculés par différence) et valeurs nutritives.

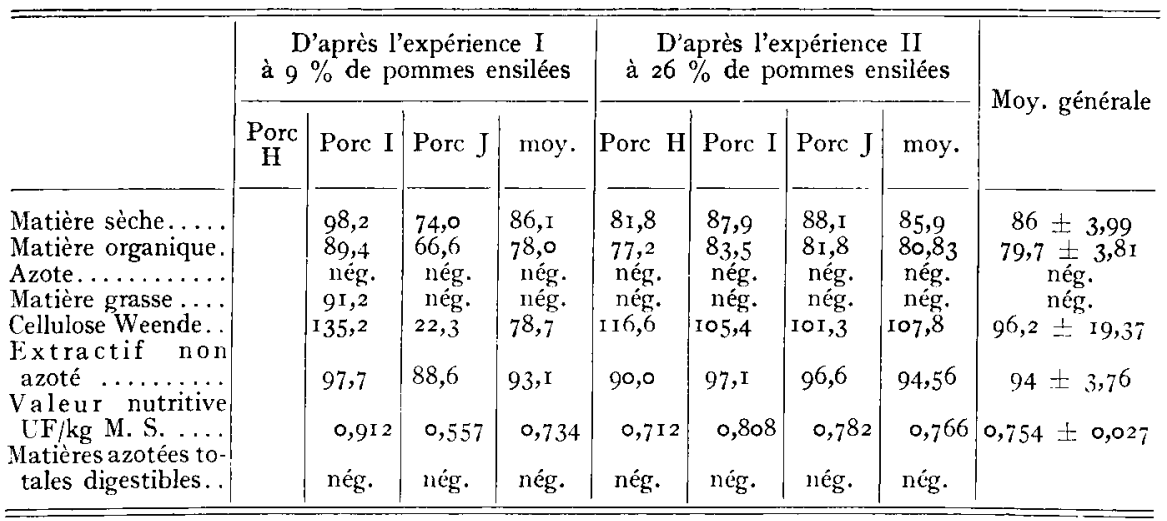


bol alimentaire à travers le tube digestif, aurait provoqué les troubles diarrhéiques signalés plus haut. La rapidité de transit pourrait donc être à l'origine de la digestibilité anormalement basse de la matière organique de pomme que nous avons observée; la forte absorption au niveau intestinal de ce même sel expliquerait le taux d'utilisation digestive illogiquement plus élevée de la matière sèche. Il est intéressant à signaler qu'une chute de digestibilité due au sel et constatée chez le mouton a été rapportée récemment par NELSON et coll. (4).

La valeur nutritive, chez le porc, de la matière sèche de l'ensilage de pommes, exempt d'alcool, calculée à partir des mesures expérimentales faites dans ces conditions, est de $0,754 \pm 0,0$ I 7 u. f. par kg pour un taux d'incorporation compris entre 9 et 26 p. Ioo. Elle n'est valable que dans le cas particulier de l'aliment expérimenté. Étant donné les perturbations de la digestibilité que nous lui attribuons, on ne saurait généraliser cette valeur à des ensilages de pommes préparés de façon différente.

\section{RÉFÉRENCES BIBLIOGRAPHIQUES}

(I) Bohstedt (G.). - Feedstuffis, I952, 24, 48, p. 26-34.

(2) Richter (K.), BECKER (M.). - Handbuch der Landw. III, I952. (Ver. Parey).

(3) Suith (W. W.). - Pork production I952 (Mc Millan New-York).

(4) Neison (A. B.), Mac Vicar (R. W.), Archer Jr (Wm) et Meiske (J. C.). - J. of Anim. Sc. I955, 14, no 3, p. 825-830. 